\title{
EQUIVALENCE BETWEEN ALMOST-GREEDY AND SEMI-GREEDY BASES
}

\author{
P. M. BERNÁ
}

\begin{abstract}
In [3] it was proved that almost-greedy and semi-greedy bases are equivalent in the context of Banach spaces with finite cotype. In this paper we show this equivalence for general Banach spaces.
\end{abstract}

\section{INTRODUCTION}

Let $(\mathbb{X},\|\cdot\|)$ be a Banach space over $\mathbb{F}(\mathbb{F}$ denotes the real field $\mathbb{R}$ or the complex field $\mathbb{C}$ ) and let $\mathscr{B}=\left(e_{n}\right)_{n=1}^{\infty}$ be a semi-normalized Schauder basis of $\mathbb{X}$ with constant $K_{b}$ and with biorthogonal functionals $\left(e_{n}^{*}\right)_{n=1}^{\infty}$, i.e, $0<\inf _{n}\left\|e_{n}\right\| \leq \sup _{n}\left\|e_{n}\right\|<\infty$ and $K_{b}=$ $\sup _{N}\left\|S_{N}(x)\right\| /\|x\|<\infty \forall x \in \mathbb{X}$, where $S_{N}(x)=\sum_{j=1}^{N} e_{j}^{*}(x) e_{j}$ denotes the algorithm of the partial sums.

As usual supp $(x)=\left\{n \in \mathbb{N}: e_{n}^{*}(x) \neq 0\right\}$, given a finite set $A \subset \mathbb{N},|A|$ denotes the cardinality of the set $A, P_{A}$ is the projection operator, that is, $P_{A}\left(\sum_{j} a_{j} e_{j}\right)=\sum_{j \in A} a_{j} e_{j}, P_{A^{c}}=\mathrm{I}_{\mathbb{X}}-P_{A}$, $\mathbf{1}_{\varepsilon A}=\sum_{n \in A} \varepsilon_{n} e_{n}$ with $\left|\varepsilon_{n}\right|=1$ (where $\varepsilon_{n}$ could be real or complex), $\mathbf{1}_{A}=\sum_{n \in A} e_{n}$ and for $A, B \subset \mathbb{N}$, we write $A<B$ if $\max _{i \in A} i<\min _{j \in B} j$.

In 1999, S. V. Konyagin and V. N. Temlyakov introduced the Thresholding Greedy Algorithm (TGA) (see [7]): given $x=\sum_{i=1}^{\infty} e_{i}^{*}(x) e_{i} \in \mathbb{X}$, we define the natural greedy ordering for $x$ as the map $\rho: \mathbb{N} \longrightarrow \mathbb{N}$ such that $\operatorname{supp}(x) \subset \rho(\mathbb{N})$ and so that if $j<k$ then either $\left|e_{\rho(j)}^{*}(x)\right|>\left|e_{\rho(k)}^{*}(x)\right|$ or $\left|e_{\rho(j)}^{*}(x)\right|=\left|e_{\rho(k)}^{*}(x)\right|$ and $\rho(j)<\rho(k)$. The $m$-th greedy sum of $x$ is

$$
\mathscr{G}_{m}(x)=\sum_{j=1}^{m} e_{\rho(j)}^{*}(x) e_{\rho(j)},
$$

and the sequence of maps $\left(\mathscr{G}_{m}\right)_{m=1}^{\infty}$ is known as the Thresholding Greedy Algorithm associated to $\mathscr{B}$ in $\mathbb{X}$. Alternatively we can write $\mathscr{G}_{m}(x)=\sum_{k \in A_{m}(x)} e_{k}^{*}(x) e_{k}$, where $A_{m}(x)=$ $\{\rho(n): n \leq m\}$ is the greedy set of $x: \min _{k \in A_{m}(x)}\left|e_{k}^{*}(x)\right| \geq \max _{k \notin A_{m}(x)}\left|e_{k}^{*}(x)\right|$.

To study the efficiency of the TGA, S. V. Konyagin and V. N. Temlyakov introduced in [7] the so called greedy bases.

Definition 1.1. We say that $\mathscr{B}$ is greedy if there exists a constant $C \geq 1$ such that

$$
\left\|x-\mathscr{G}_{m}(x)\right\| \leq C \sigma_{m}(x), \forall x \in \mathbb{X}, \forall m \in \mathbb{N},
$$

where $\sigma_{m}(x)$ is the $m$-th error of approximation with respect to $\mathscr{B}$, and it is defined as

$$
\sigma_{m}(x, \mathscr{B})_{\mathbb{X}}=\sigma_{m}(x):=\inf \left\{\left\|x-\sum_{n \in C} a_{n} e_{n}\right\|:|C|=m, a_{n} \in \mathbb{F}\right\} .
$$

2000 Mathematics Subject Classification. 46B15, 41A65.

Key words and phrases: thresholding greedy algorithm, almost-greedy bases, semi-greedy bases.

The author was supported by a PhD fellowship FPI-UAM and the grants MTM-2016-76566-P (MINECO, Spain) and 19368/PI/14 (Fundación Séneca, Región de Murcia, Spain). 
Also, S. V. Konyagin and V. N. Temlyakov characterized greedy bases in terms of unconditional bases with the additional property of being democratic, i.e, $\left\|\mathbf{1}_{A}\right\| \leq C_{d}\left\|\mathbf{1}_{B}\right\|$ for any pair of finite sets $A, B$ with $|A| \leq|B|$. Recall that a basis $\mathscr{B}$ in $\mathbb{X}$ is called unconditional if any rearrangement of the series $\sum_{n=1}^{\infty} e_{n}^{*}(x) e_{n}$ converges in norm to $x$ for any $x \in \mathbb{X}$. This turns out to be equivalent the fact that the projections $P_{A}$ are uniformly bounded on all finite sets $A$, i.e. there exists a constant $C \geq 1$ such that

$$
\left\|P_{A}(x)\right\| \leq C\|x\|, \forall x \in \mathbb{X} \text { and } \forall A \subset \mathbb{N} .
$$

Another important concept in greedy approximation theory is the notion of quasi-greedy bases introduced in [7].

Definition 1.2. We say that $\mathscr{B}$ is quasi-greedy if there exists a constant $C \geq 1$ such that

$$
\left\|x-\mathscr{G}_{m}(x)\right\| \leq C\|x\|, \forall x \in \mathbb{X}, \forall m \in \mathbb{N} .
$$

We denote by $C_{q}$ the least constant that satisfies (1) and we say that $\mathscr{B}$ is $C_{q}$-quasi-greedy.

Subsequently, P. Wojtaszczyk proved in [8] that $\mathscr{B}$ is quasi-greedy in a quasi-Banach space $\mathbb{X}$ if and only if the algorithm converges, that is,

$$
\lim _{m \rightarrow \infty}\left\|x-\mathscr{G}_{m}(x)\right\|=0, \forall x \in \mathbb{X} .
$$

One intermediate concept between greedy and quasi-greedy bases, almost-greedy bases, was introduced by S. J. Dilworth et al. in [5].

Definition 1.3. We say that $\mathscr{B}$ is almost-greedy if there exists a constant $C \geq 1$ such that

$$
\left\|x-\mathscr{G}_{m}(x)\right\| \leq C \tilde{\sigma}_{m}(x), \forall x \in \mathbb{X}, \forall m \in \mathbb{N},
$$

where $\tilde{\sigma}_{m}(x, \mathscr{B})_{\mathbb{X}}=\tilde{\sigma}_{m}(x):=\inf \left\{\left\|x-P_{A}(x)\right\|:|A|=m\right\}$. We denote by $C_{a l}$ the least constant that satisfies (2) and we say that $\mathscr{B}$ is $C_{a l}$-almost-greedy.

In [5], the authors characterized the almost-greedy bases in terms of quasi-greedy and democratic bases.

Theorem 1.4. [5, Theorem 3.3] $\mathscr{B}$ is almost-greedy if and only if $\mathscr{B}$ is quasi-greedy and democratic.

We will use the notion of super-democracy instead of democracy. This is a classical concept in this theory.

Definition 1.5. We say that $\mathscr{B}$ is super-democratic if there exists a constant $C \geq 1$ such that

$$
\left\|\mathbf{1}_{\varepsilon A}\right\| \leq C\left\|\mathbf{1}_{\eta B}\right\|
$$

for any pair of finite sets $A$ and $B$ such that $|A| \leq|B|$ and any choice $|\varepsilon|=|\eta|=1$. We denote by $C_{s d}$ the least constant that satisfies (3) and we say that $\mathscr{B}$ is $C_{s d}$-super-democratic.

Remark 1.6. It is well known that in Theorem 1.4 we can replace democracy by superdemocracy (see for instance [1, Theorem 1.3]).

On the other hand, S. J. Dilworth, N. J. Kalton and D. Kutzarova introduced in [3] the concept of semi-greedy bases. This concept was born as an enhancement of the TGA to improve the rate of convergence. To study the notion of semi-greediness, we need to define the Thresholding Chebyshev Greedy Algorithm: let $A_{m}(x)$ be the greedy set of $x$ of cardinality 
$m$. Define the $m$-th Chebyshev-greedy sum as any element $\mathscr{C}_{\mathscr{G}}{ }_{m}(x) \in \operatorname{span}\left\{e_{i}: i \in A_{m}(x)\right\}$ such that

$$
\left\|x-\mathscr{C} \mathscr{G}_{m}(x)\right\|=\min \left\{\left\|x-\sum_{n \in A_{m}(x)} a_{n} e_{n}\right\|: a_{n} \in \mathbb{F}\right\} .
$$

The collection $\left\{\mathscr{C} \mathscr{G}_{m}\right\}_{m=1}^{\infty}$ is the Thresholding Chebyshev Greedy Algorithm.

Definition 1.7. We say that $\mathscr{B}$ is semi-greedy if there exists a constant $C \geq 1$ such that

$$
\left\|x-\mathscr{C} \mathscr{G}_{m}(x)\right\| \leq C \sigma_{m}(x), \forall x \in \mathbb{X}, \forall m \in \mathbb{N} .
$$

We denote by $C_{s}$ the least constant that satisfies (4) and we say that $\mathscr{B}$ is $C_{s}$-semi-greedy.

In [3], the following theorem is proved:

Theorem 1.8. [3, Theorem 3.2] Every almost-greedy basis in a Banach space is semigreedy.

In this paper we study the converse of this theorem. In [3], the authors established the following "converse" theorem:

Theorem 1.9. [3, Theorem 3.6] Assume that $\mathscr{B}$ is a semi-greedy basis in a Banach space $\mathbb{X}$ which has finite cotype. Then, $\mathscr{B}$ is almost-greedy.

The objective here is to show that the condition of the finite cotype in the last theorem is not necessary. The main result is the following:

Theorem 1.10. Assume that $\mathscr{B}$ is a Schauder basis in a Banach space $\mathbb{X}$.

a) If $\mathscr{B}$ is $C_{q}$-quasi-greedy and $C_{s d}$-super-democratic, then $\mathscr{B}$ is $C_{s}$-semi-greedy with constant $C_{s} \leq C_{q}+4 C_{q} C_{s d}$.

b) If $\mathscr{B}$ is $C_{s}$-semi-greedy, then $\mathscr{B}$ is $C_{s d}$-super-democratic with constant $C_{s d} \leq 2\left(C_{s} K_{b}\right)^{2}$ and $C_{q}$-quasi-greedy with constant $C_{q} \leq K_{b}\left(2+3\left(K_{b} C_{s}\right)^{2}\right)$.

Remark 1.11. S. J. Dilworth et al. ([3]) proved the item a) with the bound $C_{s}=O\left(C_{q}^{2} C_{d}\right)$, where $C_{d}$ is the democracy constant. Here, we slightly relax this bound proving that $C_{s}=$ $O\left(C_{q} C_{s d}\right)$.

Corollary 1.12. If $\mathscr{B}$ is a Schauder basis in $\mathbb{X}, \mathscr{B}$ is almost-greedy if and only if $\mathscr{B}$ is semi-greedy.

\section{Preliminary Results}

To prove Theorem 1.10, we need the following technical results that we can find in [1] and [5].

\subsection{Convexity lemma.}

Lemma 2.1. [1, Lemma 2.7] For every finite set $A \subset \mathbb{N}$, we have

$$
\operatorname{co}\left\{\mathbf{1}_{\varepsilon A}:|\varepsilon|=1\right\}=\left\{\sum_{n \in A} z_{n} e_{n}:\left|z_{n}\right| \leq 1\right\},
$$

where $\operatorname{coS}=\left\{\sum_{j=1}^{n} \alpha_{j} x_{j}: x_{j} \in S, 0 \leq \alpha_{j} \leq 1, \sum_{j=1}^{n} \alpha_{j}=1, n \in \mathbb{N}\right\}$. 
As a consequence, for any finite sequence $\left(z_{n}\right)_{n \in A}$ with $z_{n} \in \mathbb{F}$ for all $n \in A$,

$$
\left\|\sum_{n \in A} z_{n} e_{n}\right\| \leq \max _{n \in A}\left|z_{n}\right| \varphi(|A|),
$$

where $\varphi(m)=\sup _{|A|=m,|\varepsilon|=1}\left\|\mathbf{1}_{\varepsilon A}\right\|$.

2.2. The truncation operator. For each $\alpha>0$, we define the truncation function of $z \in \mathbb{F}$ as

$$
T_{\alpha}(z)=\alpha \operatorname{sgn}(z),|z|>\alpha, T_{\alpha}(z)=z,|z| \leq \alpha .
$$

We can extend $T_{\alpha}$ to an operator in $\mathbb{X}$ by

$$
T_{\alpha}(x)=\sum_{i=1}^{\infty} T_{\alpha}\left(e_{i}^{*}(x)\right) e_{i}=\alpha \mathbf{1}_{\varepsilon \Gamma_{\alpha}}+P_{\Gamma_{\alpha}^{c}}(x),
$$

where $\Gamma_{\alpha}=\left\{n:\left|e_{n}^{*}(x)\right|>\alpha\right\}$ and $\varepsilon_{j}=\operatorname{sgn}\left(e_{j}^{*}(x)\right)$ with $j \in \Gamma_{\alpha}$. Hence, this is a well-defined operator for all $x \in \mathbb{X}$ since $\Gamma_{\alpha}$ is a finite set.

This operator was introduced in [3] to prove Theorem 1.8 showing that for quasi-greedy bases, this operator is uniformly bounded. A slight improvement of the boundedness constant was given in [1].

Proposition 2.2. [1, Lemma 2.5] Assume that $\mathscr{B}$ is $C_{q}$-quasi-greedy basis in a Banach space $\mathbb{X}$. Then, for every $\alpha>0$,

$$
\left\|T_{\alpha}(x)\right\| \leq C_{q}\|x\|, \forall x \in \mathbb{X} .
$$

We shall also use the following known inequality from [5].

Lemma 2.3. [5, Lemma 2.2] If $\mathscr{B}$ is a $C_{q}$-quasi-greedy basis in $\mathbb{X}$,

$$
\min _{j \in G}\left|e_{j}^{*}(x)\right|\left\|\mathbf{1}_{\varepsilon G}\right\| \leq 2 C_{q}\|x\|, \forall x \in \mathbb{X}, \forall G \text { greedy set of } x,
$$

with $\varepsilon=\left\{\operatorname{sgn}\left(e_{j}^{*}(x)\right)\right\}$.

\section{PROOF OF THE MAIN RESUlT}

Using the lemmas of Section 2 , we prove Theorem 1.10 .

Proof of Theorem 1.10 First, we show the proof of $a$ ). Suppose that $\mathscr{B}$ is $C_{q}$-quasi-greedy and $C_{s d}$-super-democratic. To show the semi-greediness, we will follow the same procedure as in the proof of [4, Theorem 4.1] and [3, Theorem 3.2]. Take $x \in \mathbb{X}$ and $z=\sum_{i \in B} a_{i} e_{i}$ with $|B|=m$ such that $\|x-z\|<\sigma_{m}(x)+\delta$, for $\delta>0$. Let $A_{m}(x)$ the greedy set of $x$ of cardinality $m$. We write $x-z:=\sum_{i=1}^{\infty} y_{i} e_{i}$, where $y_{i}=e_{i}^{*}(x)-a_{i}$ for $i \in B$ and $y_{i}=e_{i}^{*}(x)$ for $i \notin B$. To prove that $\mathscr{B}$ is semi-greedy we only have to show that there exists $w \in \mathbb{X}$ so that $\operatorname{supp}(x-w) \subset A_{m}(x)$ and $\|w\| \leq c\|x-z\|$ for some positive constant $c$. If $\alpha=$ $\max _{j \notin A_{m}(x)}\left|e_{j}^{*}(x)\right|$, we take the element $w$ as is defined in [3]:

$$
w:=\sum_{i \in A_{m}(x)} T_{\alpha}\left(y_{i}\right) e_{i}+P_{A_{m}^{c}(x)}(x)=\sum_{i=1}^{\infty} T_{\alpha}\left(y_{i}\right) e_{i}+\sum_{i \in B \backslash A_{m}(x)}\left(e_{i}^{*}(x)-T_{\alpha}\left(y_{i}\right)\right) e_{i} .
$$

Of course, $w$ satisfies that $\operatorname{supp}(x-w) \subset A_{m}(x)$ and we will prove that $\|w\| \leq\left(C_{q}+\right.$ $\left.4 C_{q} C_{s}\right)\|x-z\|$. To obtain this bound, using Proposition 2.2,

$$
\left\|\sum_{i=1}^{\infty} T_{\alpha}\left(y_{i}\right) e_{i}\right\| \leq C_{q}\|x-z\| .
$$


Taking into account that $\left|e_{i}^{*}(x)-T_{\alpha}\left(y_{i}\right)\right| \leq 2 \alpha$ for $i \in B \backslash A_{m}(x)$, using Lemma2.1, $\left\|\sum_{i \in B \backslash A_{m}(x)}\left(e_{i}^{*}(x)-T_{\alpha}\left(y_{i}\right)\right) e_{i}\right\| \leq 2 \alpha \varphi\left(\left|B \backslash A_{m}(x)\right|\right) \leq 2 \min _{j \in A_{m}(x) \backslash B}\left|e_{j}^{*}(x-z)\right| \varphi\left(\left|A_{m}(x) \backslash B\right|\right)$.

To improve the bound of $C_{s}$ as we have commented in the Remark 1.11, based on ([6, Lemma 2.1]), we can find a greedy set $\Gamma$ of $x-z$ with the following conditions:

- $|\Gamma|=\left|B \backslash A_{m}(x)\right|$,

- $\min _{j \in A_{m}(x) \backslash B}\left|e_{j}^{*}(x-z)\right| \leq \min _{j \in \Gamma}\left|e_{j}^{*}(x-z)\right|$.

Hence, using $\varepsilon=\left\{\operatorname{sgn}\left(e_{j}^{*}(x-z)\right)\right\}$ and Lemma 2.3,

$$
\min _{j \in A_{m}(x) \backslash B}\left|e_{j}^{*}(x-z)\right| \varphi\left(\left|B \backslash A_{m}(x)\right|\right) \leq C_{s d} \min _{j \in \Gamma}\left|e_{j}^{*}(x-z)\right|\left\|\mathbf{1}_{\varepsilon \Gamma}\right\| \leq 2 C_{q} C_{s d}\|x-z\| .
$$

Thus, using (6), (7), (8), the basis is $C_{s}$-semi-greedy with constant $C_{s} \leq\left(C_{q}+4 C_{q} C_{s d}\right)$.

Now, we prove $b$ ). Assume that $\mathscr{B}$ is $C_{s}$-semi-greedy.

Super-democracy can be proved using the technique of [3, Proposition 3.3]. Indeed, take $A$ and $B$ with $|A| \leq|B|$ and $|\varepsilon|=|\eta|=1$. Select now a set $D$ such that $|D|=|A|, D>(A \cup B)$ and define $z:=\mathbf{1}_{\varepsilon A}+(1+\delta) \mathbf{1}_{D}$ with $\delta>0$. It is clear that $\mathscr{G}_{|D|}(z)=(1+\delta) \mathbf{1}_{D}$. Then,

$$
\left\|z-\mathscr{C} \mathscr{G}_{|D|}(z)\right\|=\left\|\mathbf{1}_{\varepsilon A}+\sum_{i \in D} c_{i} e_{i}\right\|
$$

where the scalars $\left(c_{i}\right)_{i \in D}$ are given by the Chebyshev approximation. Then,

$$
\left\|\mathbf{1}_{\varepsilon A}\right\| \leq K_{b}\left\|\mathbf{1}_{\varepsilon A}+\sum_{i \in D} c_{i} e_{i}\right\| \leq K_{b} C_{s} \sigma_{|D|}(z) \leq K_{b} C_{s}\left\|(1+\delta) \mathbf{1}_{D}\right\|
$$

If $\delta$ goes to 0 ,

$$
\left\|\mathbf{1}_{\varepsilon A}\right\| \leq C_{S} K_{b}\left\|\mathbf{1}_{D}\right\|
$$

The next step is to obtain that $\left\|\mathbf{1}_{D}\right\| \leq 2 K_{b} C_{S}\left\|\mathbf{1}_{\eta B}\right\|$. For that, we take the element $y:=$ $(1+\boldsymbol{\delta}) \mathbf{1}_{\eta B}+\mathbf{1}_{D}$ with $\boldsymbol{\delta}>0$. Then, $\mathscr{G}_{|B|}(y)=(1+\delta) \mathbf{1}_{\eta B}$. Hence,

$$
\left\|y-\mathscr{C} \mathscr{G}_{|B|}(y)\right\|=\left\|\sum_{i \in B} d_{i} e_{i}+\mathbf{1}_{D}\right\|
$$

where as before, the scalars $\left(d_{i}\right)_{i \in B}$ are given by the Chebyshev approximation. Using again the semi-greediness,

$$
\left\|\mathbf{1}_{D}\right\| \leq 2 K_{b}\left\|\sum_{i \in B} d_{i} e_{i}+\mathbf{1}_{D}\right\| \leq 2 C_{S} K_{b} \sigma_{|B|}(y) \leq 2 C_{S} K_{b}\left\|(1+\delta) \mathbf{1}_{\eta B}\right\| .
$$

Taking $\delta \rightarrow 0$, we obtain that

$$
\left\|\mathbf{1}_{D}\right\| \leq 2 C_{s} K_{b}\left\|\mathbf{1}_{\eta B}\right\|
$$

Using (9) and (10),

$$
\left\|\mathbf{1}_{\varepsilon A}\right\| \leq 2\left(C_{S} K_{b}\right)^{2}\left\|\mathbf{1}_{\eta B}\right\| .
$$

Hence, the basis is super-democratic with constant $C_{s d} \leq 2\left(C_{s} K_{b}\right)^{2}$.

To prove now the quasi-greediness, we will present a more elemental proof than in [3, Theorem 3.6] that works for general Banach spaces: take an element $x \in \mathbb{X}$ with finite 
support and $A_{m}(x)$ the greedy set of $x$ with cardinality $m$, take $D>\operatorname{supp}(x)$ with $|D|=$ $\left|A_{m}(x)\right|=m$ and define $z:=x-\mathscr{G}_{m}(x)+(\boldsymbol{\delta}+\alpha) \mathbf{1}_{D}$, where $\delta>0$ and $\alpha=\min _{j \in A_{m}(x)}\left|e_{j}^{*}(x)\right|$. Then, since $A_{m}(z)=D$,

$$
\left\|z-\mathscr{C} \mathscr{G}_{m}(z)\right\|=\left\|x-\mathscr{G}_{m}(x)+\sum_{i \in D} f_{i} e_{i}\right\|,
$$

for some scalars $\left(f_{i}\right)_{i \in D}$ given by the Chebyshev approximation. Then,

$$
\left\|x-\mathscr{G}_{m}(x)\right\| \leq K_{b}\left\|x-\mathscr{G}_{m}(x)+\sum_{i \in D} f_{i} e_{i}\right\| \leq K_{b} C_{s} \sigma_{m}(z) \leq K_{b} C_{s}\left\|x+(\delta+\alpha) \mathbf{1}_{D}\right\| .
$$

Taking $\delta \rightarrow 0$,

$$
\left\|x-\mathscr{G}_{m}(x)\right\| \leq K_{b} C_{s}\left\|x+\alpha \mathbf{1}_{D}\right\| \leq K_{b} C_{S}\left(\|x\|+\left\|\alpha \mathbf{1}_{D}\right\|\right) .
$$

Select now $y:=\sum_{j \in A_{m}(x)}\left(e_{j}^{*}(x)+\delta \varepsilon_{j}\right) e_{j}+\sum_{j \in A_{m}^{c}(x)} e_{j}^{*}(x) e_{j}+\alpha \mathbf{1}_{D}$, with $\delta>0$ and $\varepsilon_{j}=$ $\operatorname{sgn}\left(e_{j}^{*}(x)\right)$ for $j \in A_{m}(x)$. Then, since $\mathscr{G}_{m}(y)=\sum_{j \in A_{m}(x)}\left(e_{j}^{*}(x)+\delta \varepsilon_{j}\right) e_{j}$, using Chebyshev approximation,

$$
\left\|y-\mathscr{C} \mathscr{G}_{m}(y)\right\|=\left\|\sum_{j \in A_{m}(x)} a_{i} e_{i}+\sum_{j \in A_{m}^{c}(x)} e_{j}^{*}(x) e_{j}+\alpha \mathbf{1}_{D}\right\| .
$$

Hence,

$$
\begin{aligned}
\left\|\alpha \mathbf{1}_{D}\right\| & \leq 2 K_{b}\left\|\sum_{j \in A_{m}(x)} a_{i} e_{i}+\sum_{j \in A_{m}^{c}(x)} e_{j}^{*}(x) e_{j}+\alpha \mathbf{1}_{D}\right\| \leq 2 K_{b} C_{s} \sigma_{m}(y) \\
& \leq 2 K_{b} C_{S}\left\|\sum_{j \in A_{m}(x)}\left(e_{j}^{*}(x)+\delta \varepsilon_{j}\right) e_{j}+\sum_{j \in A_{m}^{c}(x)} e_{j}^{*}(x) e_{j}\right\| .
\end{aligned}
$$

Taking $\delta \rightarrow 0,\left\|\alpha \mathbf{1}_{D}\right\| \leq 2 K_{b} C_{S}\|x\|$. Using the last inequality and (11),

$$
\left\|x-\mathscr{G}_{m}(x)\right\| \leq K_{b} C_{s}\left(\|x\|+2 K_{b} C_{s}\|x\|\right) \leq 3\left(K_{b} C_{s}\right)^{2}\|x\| .
$$

Thus, $\left\|x-\mathscr{G}_{m}(x)\right\| \leq 3\left(K_{b} C_{s}\right)^{2}\|x\|$ for any finite $x \in \mathbb{X}$ and $m \leq|\operatorname{supp}(x)|$.

For the general case, we take $x \in \mathbb{X}$ and $A_{m}(x)$ the greedy set of $x$ with cardinality $m$. We can find a number $N \in \mathbb{N}$ such that $A_{m}(x) \subset\{1, \ldots, N\}$. Then, since $\mathscr{G}_{m}(x)=\mathscr{G}_{m}\left(S_{N}(x)\right)$, applying that $\mathscr{B}$ is Schauder and quasi-greedy for elements with finite support,

$$
\begin{aligned}
\left\|x-\mathscr{G}_{m}(x)\right\| & \leq\left\|x-S_{N}(x)\right\|+\left\|S_{N}(x)-\mathscr{G}_{m}(x)\right\| \\
& =\left\|x-S_{N}(x)\right\|+\left\|S_{N}(x)-\mathscr{G}_{m}\left(S_{N}(x)\right)\right\| \\
& \leq 2 K_{b}\|x\|+3\left(K_{b} C_{s}\right)^{2}\left\|S_{N}(x)\right\| \\
& \leq K_{b}\left(2+3\left(K_{b} C_{s}\right)^{2}\right)\|x\| .
\end{aligned}
$$

This completes the proof.

Proof of Corollary 1.12 : The proof follows using Theorem 1.10, Theorem 1.4 and Remark 1.6. 
Remark 3.1. In [2, Section 6-Question 3], the authors ask the following question: if a basis $\mathscr{B}$ satisfies Property (A) and the inequality (5), is $\mathscr{B}$ semi-greedy? We remind that $\mathscr{B}$ satisfies Property $(A)$ if there is a positive constant $C_{a}$ such that

$$
\left\|x+\mathbf{1}_{\varepsilon A}\right\| \leq C_{a}\left\|x+\mathbf{1}_{\eta B}\right\|,
$$

for any $x \in \mathbb{X}, A, B$ such that $|A|=|B|<\infty, A \cap B=\emptyset,(A \cup B) \cap \operatorname{supp}(x)=\emptyset,|\varepsilon|=|\eta|=1$ and $\max _{j}\left|e_{j}^{*}(x)\right| \leq 1$. The answer is not due to the example in [1, Subsection 5.5] of a basis $\mathscr{B}$ in a Banach space such that $\mathscr{B}$ satisfies the Property (A) and (5), but is not quasi-greedy, hence is not almost-greedy and using Theorem $1.10 \mathscr{B}$ is not semi-greedy.

\section{OPEN QUESTIONS}

As discussed in [8] (see also [4]), one can define the Thresholding Greedy Algorithm and the Thresholding Chebyshev Greedy Algorithm in the context of Markushevich bases, that is, $\left\{e_{i}, e_{i}^{*}\right\}$ is a semi-normalized biorthogonal system, $\left.\mathbb{X}=\overline{\operatorname{span}\left\{e_{i}: i \in \mathbb{N}\right.}\right\}$ and $\mathbb{X}^{*}=$ $\overline{\operatorname{span}\left\{e_{i}^{*}: i \in \mathbb{N}\right\}}{ }^{w^{*}}$. In section a) of Theorem 1.10, it is enough to work with Markushevich bases instead of Schauder bases. However, in the item b), seems to be necessarily to use that $\mathscr{B}$ is Schauder to prove the result.

Question 1: Is it possible to remove the condition to be Schauder in section b) of Theorem 1.10 ?

Another interesting problem is to establish if almost-greediness implies the condition to be Schauder. Of course, if $\mathscr{B}$ is greedy then $\mathscr{B}$ is Schauder since greediness implies unconditionality. As far as we know, all of examples of almost-greedy bases in the literature seem to be Schauder bases, but we don't know if almost-greediness implies that $\mathscr{B}$ is Schauder or not.

Question 2: If $\mathscr{B}$ is almost-greedy, is it necessarily Schauder?

Acknowledgments: Thanks to Eugenio Hernández, Gustavo Garrigós, Fernando Albiac and José Luis Ansorena for many interesting discussions during the elaboration of this paper.

\section{REFERENCES}

[1] P. M. BERnÁ, Ó. Blasco, G. GARRIGós, Lebesgue inequalities for greedy algorithm in general bases, Rev. Mat. Complut. 30, 369-392 (2017).

[2] P. M. Berná, S. J. Dilworth, D. Kutzarova, T. Oikhberg, B. Wallis, The weighted Property (A) and the greedy algorithm (Submitted) https://arxiv.org/abs/1803.05052.

[3] S. J. Dilworth, N. J. Kalton, D. Kutzarova, On the existence of almost greedy bases in Banach spaces, Studia Math. 159 (2003), 67-101.

[4] S. J. Dilworth, D. Kutzarova, T. OikHBERG, Lebesgue constants for the weak greedy algorithm, Rev. Matem. Compl. 28(2), 393-409 (2015).

[5] S. J. Dilworth, N. J. Kalton, D. Kutzarova, V. N. Temlyakov, The thresholding greedy algorithm, greedy bases, and duality, Constr.Approx. 19 (2003), no.4, 575-597.

[6] G. Garrigós, E. Hernández, T. OIKHBERG, Lebesgue-type inequalities for quasi-greedy bases, Constr. Approx. 38 (2013), 447-470.

[7] S. V. Konyagin, V. N. Temlyakov, A remark on greedy approximation in Banach spaces, East J. Approx. 5 (1999), 365-379.

[8] P. WojtAszczyк, Greedy algorithm for general biorthogonal systems, J.Approx.Theory 107 (2000), no.2, 293-314. 
Pablo M. Berná, Departmento de Matemáticas, Universidad Autónoma de Madrid, 28049 MADRID, SPAIN

E-mail address: pablo.berna@uam.es 